\title{
Research on the realization of 3D urban traffic simulation system
}

\author{
ZhongHua Caoํㅜ, QuanHua Tang, YuangChun Liu
}

\begin{abstract}
This paper makes an intensive study of the realizations and related key technologies in the 3D urban traffic simulation system. First, the system can quickly edit the scenes of the 3D urban, including buildings, roads, etc. It describes the roads using the graph structure, then, the constrained Delaunay algorithm is applied to reconstruct the mesh of the road and terrain. The most important, the system realizes the intelligent automatic movement of the vehicles. The technologies of LOD, back-face culling, navigation points, B-tree and so on are introduced to improve the simulation efficiency. Thus it can be used to simulate the traffic, plan the design of urban road.
\end{abstract}

Keywords: 3D urban $\cdot$ traffic simulation $\cdot$ Delaunay algorithm $\cdot$ navigation points $•$ B-tree

\section{Introduction}

Traffic simulation is an important application of computer technology in the field of transport. The engineers or researchers can use it to test and optimize the design of the roads. Modern urban transport system is increasingly complex. So the software should be an intuitive, convenient, flexible traffic analysis tools to achieve the 3D representation of the urban traffic scene, highly intuitive performance of the road network congestion and fluid situation, providing technical basis for traffic road design, comparing and evaluating various parameters [1][2][3].

For a modern three-dimensional traffic simulation system, it need not only provides building the urban scenes, transport network through the relevant data, and also provide intelligent module for real-time traffic simulation on the road. So the road network should be relatively independent of the terrain, and many technologies are used to optimize the simulation effect. The following paper mainly describes those contents.

\footnotetext{
${ }^{1}$ ZhongHua Cao( $\left.\square\right)$

School of Software, JiangXi Normal University.330022,NanChang, China

e-mail: boyangczh@sohu.com. supported by 3208
} 


\section{System Structure}

The system is mainly divided into five modules: create terrain, quickly construct the scene of any urban, edit a variety of network of roads manually, real-time traffic simulation and data analysis.

Terrain creation: Terrain is the carrier of the entire virtual transport network. It is closely related to other objects in the system. There are a variety of ways to achieve the init terrain, such as using gray scale image, DEM data and arbitrary triangle terrain.

Edit the road network: There are three different types of roads: straight, curve and cross roads. The system can complete manually editing different types of roads, including moving, rotating, scaling and so on, then achieve the overall construction of the road network.

Construct the scenes: Any of the three-dimensional scenes can be quickly construct by loading three-dimensional models. It supports some well-known format, such as 3ds, obj. In addition, the system also offers two special scene effects - rain and fog.

Traffic simulation: In the Traffic simulation, first, the system uses navigation point techniques in order to reduce calculating time, vehicle automatically run on the road according to the points. Then according to the view of frustum, the vehicles can achieve a variety of behaviours, such as acceleration, deceleration, overtaking, and finally realize the induction of traffic light signal, namely you stop at a red light, the green line. System support three observation ways: manual navigation, bird's eye view and vehicle tracking. Through these ways, it can see any one corner of the $3 \mathrm{~d}$ urban, and then more clearly understanding the city's traffic condition.

Data analysis: Compute and analysis the vehicle's total flow, peak and valley value, the amount of accidents and other information on each road. All the information will be presented in form.

\section{The Core Technique of the System}

\subsection{Road's Data Format}

In real life, the road network is composed of two basis elements: intersections and road segments. It can be descript by the graph structure, intersections as nodes and roads as edges. Intersections of the road can be turning, fork or cross, which was reflected in the graph structure. In this system, the road data is divided into road segments and road nodes. They are stored in file formats such as shown in Table 1 and Table 2. 
Table 1 Road Segment Data Format

\begin{tabular}{|c|c|c|r|r|r|}
\hline road ID & start ID of road node & end ID of road node & width & one-way & texture \\
\hline 1 & 1 & 2 & 2 & 1 & 1 \\
\hline 2 & 2 & 3 & 2 & 1 & 2 \\
\hline$\ldots$ & & & & & \\
\hline
\end{tabular}

Table 2 Road Node Data Format

\begin{tabular}{|r|c|c|c|c|c|}
\hline $\begin{array}{l}\text { node } \\
\text { ID }\end{array}$ & x coordinate & y coordinate & $z$ coordinate & node degree & relevant edges ID \\
\hline 1 & 1009 & 0 & 12 & 1 & 1 \\
\hline 2 & 5 & 0 & -2 & 3 & 1213 \\
\hline$\ldots$ & & & & & \\
\hline
\end{tabular}

\subsection{Road and Terrain Matching Solution}

The matching of road and terrain is in essence to reconstruct the terrain by the road data. In order to easily operate and extract the road information, the road network must be independent of the terrain. The reconstruction of terrain mesh is realized by using Delaunay algorithm. Here the system mainly consider the generation of straight line, corner, cross roads, and how to join together between them. The steps are shown below.

For road reconstruction, it must first get a closed road region in the terrain. When the type and parameters of road is set, then the enclosed region can be determined by finding the intersections of terrain and road, which are lie in the four borders of the road segment. The second step is to remove points of the terrain within the closed region, then an empty and enclosed region, which will be used to construct road, is got. At last, the Delaunay algorithm realizes the triangular mesh reconstruction in the road interior and the terrain near the external road border. Both are rendered independently [1][2][3].

\subsubsection{Construct scenes}

This module provides the interface to load model, which can be 3ds and obj files. Users can select the appropriate model according to the picture in the interface, and operate each model in the scene, such as delete, move, rotate, zoom. All modules are linked using a list structure, where those operations are recorded.

\subsubsection{Road edit and graph generated}


Visual editing the road also includes the functions of moving, rotating, deleting, and so on. In order to improve system efficiency, first, roads are created above the surface of terrain. If all works are confirmed, the system will complete the matching of road and terrain, and generate the graph, which include the data of road segments and road nodes.

Due to road represented by many segments, the system must consider how to join them together. In the 3D world, it is too complex to manually operate the road in order to perfectly splice two adjacency segments. Therefore, an automatic technique is need, which joining the adjacency road together. At last Users can finetune the corresponding data. The final result of the scenes is shown Fig 1.

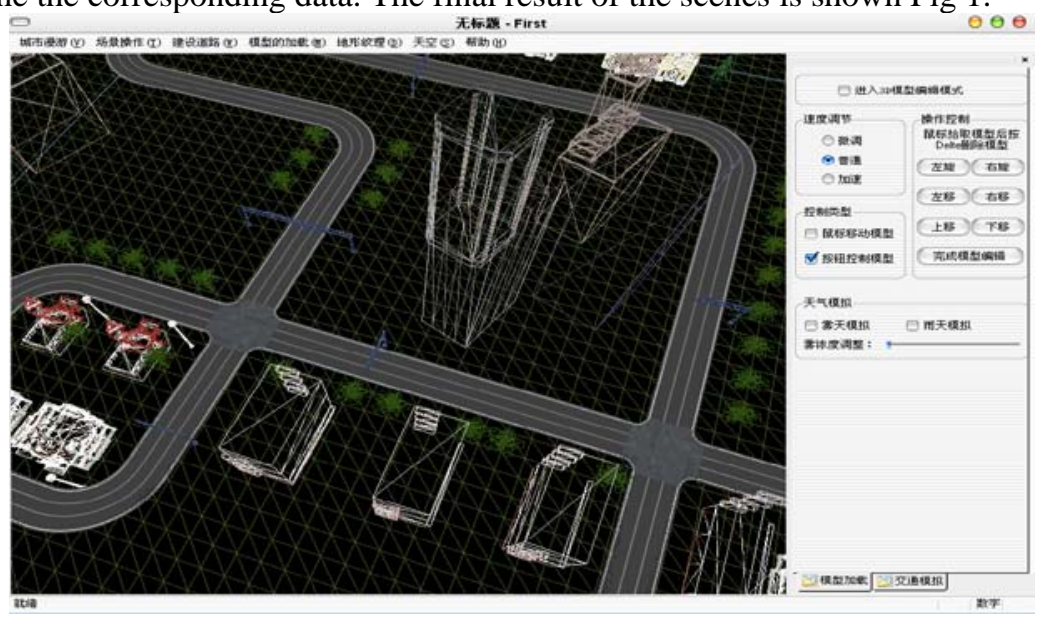

Fig 1 The urban scene after completing its construction

\subsection{Traffic Simulation}

There are thousands of objects in the 3D urban. It is very difficult for the computer to render and compute them, even if you have good hardware devices. This requires using efficient algorithms to optimize it, such as back-face culling algorithm, viewing frustum clipping algorithm. It is more important to implement simulating many vehicles running on the road. Traffic simulation includes vehicle automatically intelligent driving, manual navigation, traffic statistics and picking up the vehicle for tracking. It is clear that the realization of the vehicles' movement should be considerate first. Vehicles move on the road, it is mean the coordinate of the car is changing in consonance with the road coordinate [4][5][6].

\subsubsection{Dynamic running based on priority and time access list}


In order to achieve vehicles dynamic movement, you need to accurately calculate the time. It is the key issues in the traffic movement simulation. The system uses two kinds of timer: delay timer and Window message timer-WM_TIMER. Each car object has a function OnAnimate(float deltaTime), the input parameters returned by the function GetElapsed (), is the total time elapsed since the last function called. The new position of the car is calculated here.

When there are many vehicles, the system will be busy in calculating all kinds of data. There will inevitably appear discontinuous problems in the movement of vehicles. It maintains a vehicle link chain, and gives every car corresponding priority. Objects in the viewing frustum, will be calculated and rendered first, have higher priority than objects outside. Those will be rendered by independent thread. Traffic lights timer control uses a new WM_TIMER timer message.

\subsubsection{Intelligent moving based on navigation points}

In order to enhance the speed of process, multithread and LOD technology has been adopted. Vehicles in visible and invisible field of vision are submitted to different threads to process. The LOD algorithm is used to render models with different details in the field of vision.

Navigation points are a series of points that are generated together with the editing of roads, saved in the scene information and distribute regularity. The routing path is based on navigation points, and vehicles change the coordinate in accordance with the graph edge. The vehicles intelligently drive along the navigation path. It will search the corresponding points before entering each road, dynamically change the direction of movement through the function of Find_Nearest_Waypoint. The idea is shown by the following Fig 2.

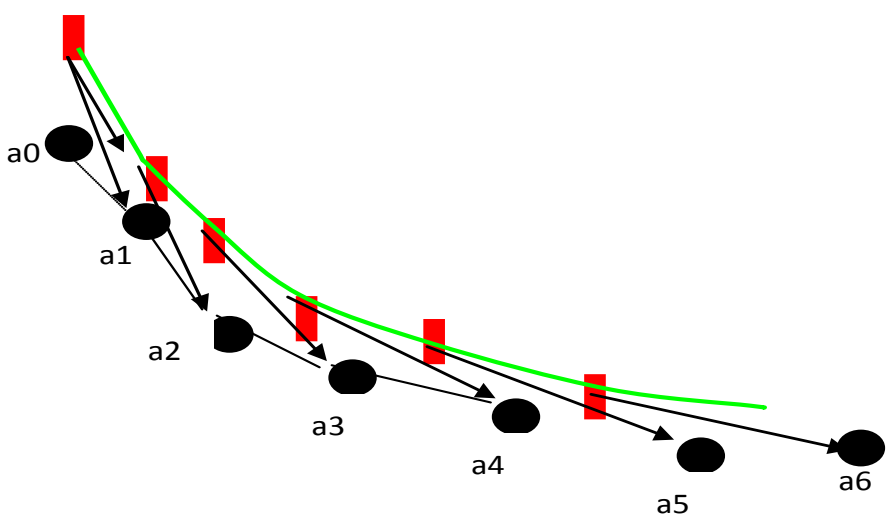

Fig 2 Red block represents car, black dot represents the navigation point. The blue trajectory represents the moving track in the curves. 


\subsubsection{Vehicles avoidance strategy}

When moving on road, vehicle will encounter all kinds of circumstances, such as following driving, overtaking, etc. Each vehicle based on its own field of vision, will search other vehicles nearby, and decide how to do according to predetermined safe distance, coordinates, speed, etc. For example, if the vehicle will collide with other vehicle, it should decide to change road to overtake or decelerate to follow.

To increase search speed, the system limit the searching scopes are around current and adjacent roads. A B-tree is established, road as a child node, vehicles as the leaf node, to save the information about vehicles on roads [7]. Vehicles in the boundary of the driveway connection or the place having traffic light, should be kept to following driving in sequence.

If the current car and the preceding car meet the relationship of overtaking conditions, it can switch to an adjacent driveway to overtake. The realization of overtaking is switching the channel between the different navigation points.

\subsubsection{Simulation results}

The system is implemented on visual studio 2010 development platform by using $\mathrm{C}++^{+}$and OPENGL [8]. It has been tested under the following environment: I5-3210, 4g ram, NVIDIA GT635M. The simulation results are as follows in table 3.

Table 3 simulation results

\begin{tabular}{|l|l|l|}
\hline & Number of cars & Fps \\
\hline simulation before optimization & 200 & 26 \\
\hline simulation after optimization & 1000 & 50 \\
\hline
\end{tabular}

\section{Conclusions}

The 3D urban traffic simulation is a complicated, comprehensive system. It must process a large number of data to meet vision effect and simulation application needs. This paper introduces the structure of the system in detail, puts forward some problems, which should be solved and the solutions. By using these technologies mentioned above, the system can simulate thousands of vehicles at the same time, and maintain running well. The next step of the system will focus on how to improve vehicle's intelligent behavior and analysis of traffic accidents, so it can get more accurate and realistic results in the simulation. 


\section{References}

1. Wang Fujian, Wu Guoxiong(2004). 3d modeling technology of road engineering. China Communications Press.

2. Tingkai Wang, Shanyu Tang, Peiyuan Pang.3D urban traffic system simulation based on geodata[J].IEEE 2nd International Conference on Information Technology:Research and Education, pp.59-63,2004.

3. Pu Hao, Song Zhangfeng. 3D-modelling for roads based on constrained Delaunay triangulation, Huazhong University of science and technology press (natural science). 33(6), pp.111113, 2005.

4. You Feng. Study on Autonomous Lane Changing and Autonomous Overtaking Control Method of Intelligent Vehicle[D], ChangChun:JiLin University, 2005.

5. Kosmatopoulos E.B,Ioannou P.A.Collision avoidance analysis for lane changing and merging[J].IEEE Transactions on Vehicular TechnoloLogy, 49(6),pp.2295-2308,2000.

6. Gao Feng, Yang XinHong, Xu GuoYanm, Virtual Realization of Active Avoiding Collision of Vehicles’ Intelligent Running, Chinese Journal of Mechanical Engineering,45(8),pp.203-209, 2009.

7. Sartaj Sahni(2000). Data Structures Algorithms and Applications in C++ BeiJing: Machinery Industry Press.

8. Richard S.Wright,Jr.Benjamin Lipchak(2010).OpenGL SuperBile,4th Edition BeiJing: People's posts and Telecommunications Press. 